\title{
Time reversal reconstruction of finite sized sources in elastic media
}

\author{
Brian E. Anderson \\ Acoustics Research Group, Department of Physics and Astronomy, Brigham Young University, N283 Eyring \\ Science Center, Provo, Utah 84602 \\ bea@byu.edu \\ Michele Griffa \\ Laboratory for Building Science and Technology, EMPA, Swiss Federal Laboratories for Material Science and \\ Technology, CH-8600, Dübendorf, Switzerland \\ michele.griffa@empa.ch \\ Timothy J. Ulrich and Paul A. Johnson \\ Solid Earth Geophysics Group (EES-17), Los Alamos National Laboratory, Los Alamos, New Mexico 87545 \\ tju@lanl.gov,paj@lanl.gov
}

\begin{abstract}
The ability of the time reversal process to reconstruct sources of finite size relative to a wavelength is investigated. Specifically the quality of the spatial reconstruction of a finite sized source will be presented through the use of time reversal experiments conducted on an aluminum plate. The data presented in the paper show that time reversal can reconstruct a source equally well regarding less of its size, when the source is a half wavelength or less in size. The quality of spatial reconstruction when the source is larger than a half wavelength progressively decreases with the size of the source.
\end{abstract}

(C) 2011 Acoustical Society of America

PACS numbers: 43.60.Tj, 43.60.Lq, 43.40.Le, 43.40.Ph [CG]

Date Received: July 29, $2011 \quad$ Date Accepted: August 16, 2011

\section{Introduction}

Time reversal (TR) is a method of focusing wave energy, temporally and spatially, onto a specific location. ${ }^{1,2}$ One of the purposes of TR is to reconstruct an unknown source both temporally and spatially in order to locate it and characterize it. TR in solid media has found application in nondestructive evaluation, to localize and characterize cracks in mechanical parts, ${ }^{3,4}$ to estimate shear elasticity of materials, ${ }^{5}$ to locate landmines, ${ }^{6}$ and for imaging of human teeth. ${ }^{7}$ It has also found application in the localization of seismic events ${ }^{8,9}$ and localization in touch panel applications. ${ }^{10,11}$

TR generally consists of two phases. The first phase is the forward propagation in which a collection of receivers, referred to as the time reversal mirror (TRM), records the incident wave energy produced by some source. In the second phase, the received signals are flipped in time and broadcast from the TRM (where receivers now act as sources), and portions of the emitted wave energy traverses the forward propagation paths to reconstruct the source(s) which originally started the forward propagation.

Larmat et al. showed that the rupture evolution of the great Sumatra earthquake in the year 2004 could be reconstructed with TR, providing evidence that sources of finite size could be reconstructed. ${ }^{8}$ However, this did not answer the question of whether a coherent finite sized source (all parts of the source emitting simultaneously) could be reconstructed with TR.

The effects of the finite size of a transducer used in a TRM on the TR process has been observed previously by Griffa et al., ${ }^{12}$ though this was not the focus of their work. Their work presented observations from two 2D numerical examples that employed a single channel TRM transducer of two different lengths $d_{1} / \lambda=1.69$, and 
$d_{2} / \lambda=0.18$, where $d$ is the transducer length and $\lambda$ is the wavelength. Their results showed that the spatial focus was easier to distinguish with the smaller TRM transducer.

Later, Kremers et al. studied the effects of finite sized sources when using TR in seismic applications. ${ }^{13}$ They showed that three double-couple point sources, separated in time, and in space on the order of a wavelength could also be imaged correctly. They then studied the ability of TR to reconstruct the finite rupture process of the Japan Tottori earthquake in the year 2000 and found that the desired details of the rupture were lost. They concluded that only point sources could be resolved by TR. However, they admitted that their numerical experiments were not exhaustive on this subject. Further, Larmat et al. had already shown that the rupture process could be imaged. ${ }^{8}$ The difference may lie in the fact that the work of Larmat et al. was conducted in the entire Earth "cavity" whereas the work of Kremers et al. was strictly on a regional scale. It should also be pointed out that, in relation to the present study, neither Kremers et al. nor Larmat et al. studied coherent sources of finite size as their studies focused on the time evolution of rupture processes of earthquakes.

The ability of TR to reconstruct coherent sources of finite size, with respect to a wavelength, has thus not been exhaustively studied. The purpose of this paper is to fill in that gap by presenting a quantitative experimental study aimed at determining the ability of the TR process to reconstruct coherent sources of finite size. A series of 25 coherent point sources, spaced $\lambda / 16$ apart, arranged as a line source are used here in a 2D plate sample to study the quality of TR reconstruction of sources of finite size. The results show that the size of the source does not affect TR reconstruction provided that the source is a half wavelength or less, but that it does steadily degrade as the size of the source is larger than a half wavelength. Since all real sources are of finite size, the implication of this finding is that there is an upper frequency limit to the quality of TR reconstruction.

\section{Experiment}

Consider a medium in which two transducers are located at positions $S$ and $R$. A source is emitted from the transducer located at $S$ and the forward propagation is recorded by the transducer at $R$, and this recorded signal is then reversed in time. If one uses a laser vibrometer to detect the forward propagation at $R$ then the laser vibrometer itself cannot be used to broadcast this time reversed signal. Instead, the time reversed signal may be broadcast by the original source transducer, and due to reciprocity, a focusing of energy should occur at the position of the laser vibrometer. With this configuration, it is as if a virtual source has been created at the point where the laser vibrometer is focused, since a TR focus is a reconstruction of the source. This method is termed the virtual source method (VSM). ${ }^{14}$ In this manner, a virtual source may be placed at any location on the surface of a solid sample. The source transducer is then considered to be the TRM. When a multiple element TRM is used, the source signal is broadcast from each TRM element individually and the forward propagation signal is detected at a desired virtual source position. Then each forward propagation signal is time reversed and the signals may be simultaneously broadcast from each appropriate element within the TRM and the TR focus is then enhanced due to the use of multiple elements. Accordingly, it is as if the virtual source (at the laser position) emitted the various forward propagation signals.

In each measurement presented in this paper, electronic sine wave voltage signals modulated by a sine squared envelope, $V(t)$, are sent to the source transducer where

$$
V(t)=A \cos ^{2}\left[\frac{\pi\left(t-\frac{T}{2}\right)}{2 P W}\right] \cos \left[2 \pi f\left(t-\frac{T}{2}\right)\right], \text { for } \frac{T}{2}-P W \leq t \leq \frac{T}{2}+P W, \text { else } V(t)=0
$$


where $A$ is an arbitrary user-defined voltage magnitude, $T$ is the total signal length, $P W$ is the pulse width (defined as the width at half of the peak magnitude), $t$ is time, and $f$ is the sine wave frequency.

An experiment is conducted to determine the effect on focal quality of a TR process due to the size of the virtual source used. This experiment is conducted on an aluminum plate sample which measures $5.0 \mathrm{~mm}$ by $104 \mathrm{~mm}$ by $197 \mathrm{~mm}$ in size. The wave propagation in the plate is dominated by flexural waves with a speed of sound of

$$
c_{B}=\sqrt{\omega \sqrt{\frac{E h^{2}}{12 \rho\left(1-\sigma^{2}\right)}}}
$$

where $\omega$ is the angular frequency (radians per second), $E$ is the Young's modulus of elasticity, $h$ is the plate thickness, $\rho$ is the material density, and $\sigma$ is the Poisson's ratio. This gives $c_{B}=3140 \mathrm{~m} / \mathrm{s}$ (assuming $\omega=1257 \mathrm{krad} / \mathrm{s}, E=71 \mathrm{GPa}, \rho=2700 \mathrm{~kg} / \mathrm{m}^{3}$, and $\sigma=0.33$ ). The source used in this experiment is a sine wave pulse centered at 200 $\mathrm{kHz}$ with a $10 \mu \mathrm{s} \mathrm{PW}$. The plate has eight $1 \mathrm{MHz}$ (thickness resonance frequency) PZT-5H transducers (2 mm thick, and $12.7 \mathrm{~mm}$ in diameter) distributed somewhat randomly along three sides of the plate. Figure 1 displays a photograph of the sample used in this study. These transducers are found to operate more efficiently at $200 \mathrm{kHz}$ rather than at $1 \mathrm{MHz}$ in solid samples due to a radial resonance mode of these disk transducers.

A series of virtual source experiments are conducted, using the previously described VSM, with the laser positioned on the side of the plate with no bonded transducers (see Fig. 1). In total twenty five virtual source experiments are conducted along a line parallel to the edge of the plate with $1 \mathrm{~mm}$ spacing $(\lambda=15.7 \mathrm{~mm}$, thus the spacing is equivalent to nearly a sixteenth of a wavelength) between each virtual source. The experiments conducted for this paper utilize a sampling frequency of 10 $\mathrm{MHz}$ and signal lengths of 32768 points or about $T=3.28 \mathrm{~ms}$. When conducting TR experiments, the center of the source pulse in time is set to be the center of the $3.28 \mathrm{~ms}$ time window, giving 1.64 seconds of available data time to collect the direct arrival and multiple received reflections. The focal signals are naturally expected to be centered in this same time window. See Figs. 2(a)-2(d) for typical temporal signals at

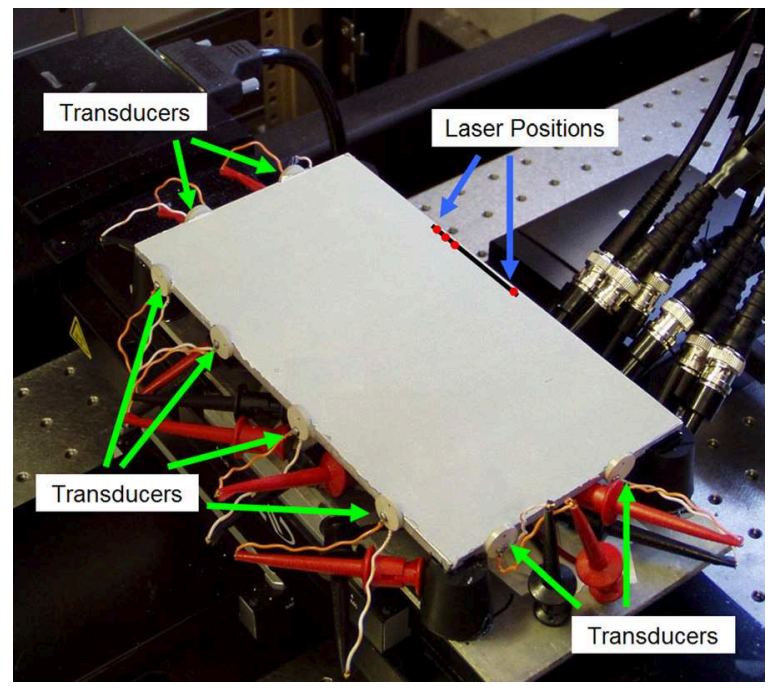

Fig. 1. (Color online) Photograph of the aluminum plate sample used in the experiments presented in this paper. The piezoelectric transducers are denoted by the green arrows, while the virtual source laser positions, along the black line on the plate, are denoted by the red dots on the plate and the blue arrows pointing to them. 
each stage of the TR process. After the forward propagation experiments are conducted, the broadcast of the time reversed signals is measured by a laser vibrometer with the plate in a scanning configuration. The TR broadcast is repeated with the laser vibrometer step-wise positioned at $1 \mathrm{~mm}$ increments over a grid covering the entire surface of the plate (with 144 averages taken at each position). Thus the spatial and temporal evolution of the focus creation can be monitored for the entire plate surface.

Figures 2(e)-2(f) display typical forward and TR focal wave fields for a single virtual source experiment. Four metrics are computed for evaluating the quality of the foci, from a snapshot in time (at the peak focal time) of each time reversed focal wave

(a)

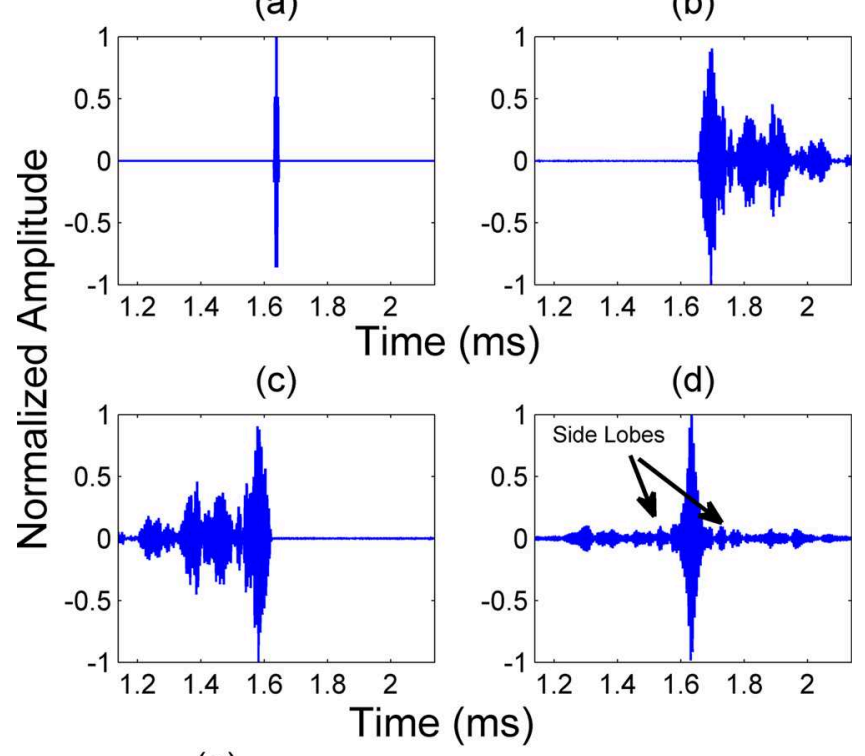

(e)

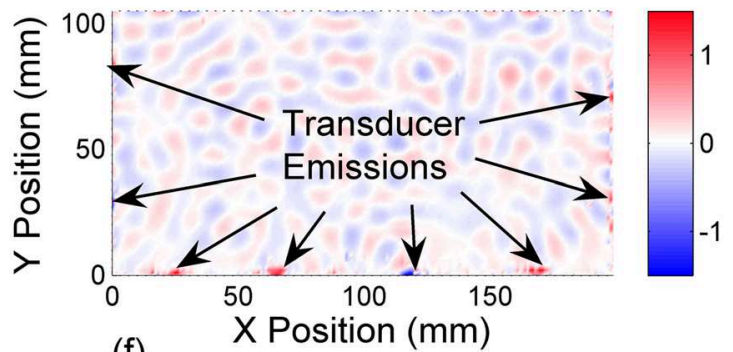

(f)

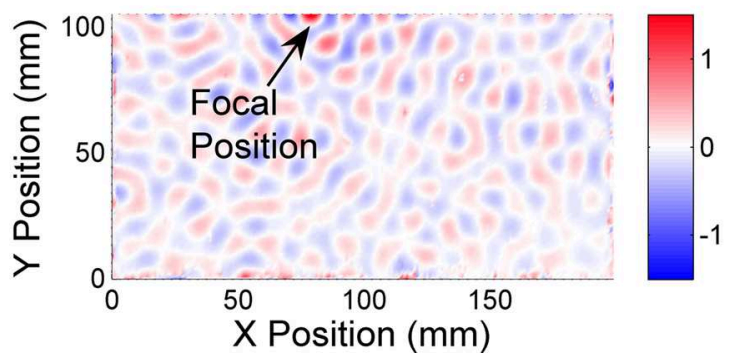

Fig. 2. (Color online) (a)-(d) are sample waveforms used in the time reversal (TR) experiments. (a) Sample source waveform. (b) Sample signal detected at virtual source position during forward propagation. (c) Reversed version of (b). (d) Sample TR focus signal detected at the virtual source position. (e) and (f) Snapshots in time of the TR out-of-plane velocity wave field prior to the focal time and at the focal time, respectively. 
field. The first metric, $\alpha$, is the peak magnitude of the spatially distributed TR focus. The second metric, $\beta$, is the ratio of $\alpha$ to the next highest spatial peak in a plot of the magnitude of the wave field. The third metric, $\gamma$, is the ratio of $\alpha$ to the average magnitude of the spatial wave field, not including the main focal lobe. The second and third metrics essentially give an idea of how easy it is for one to distinguish a focus from noise. In the post processing phase, adjacent virtual source temporal signals may be summed to effectively form a line source of finite size up to $24 \mathrm{~mm}$ in length or roughly 1.5 wavelengths. For these extended virtual sources, each of the temporal focal signals at each virtual source location are summed together to simulate the spatial averaging of a transducer when it senses an incident wave field impinging on it. The fourth metric, $\delta$, is the ratio of the peak focal magnitude of this summed temporal signal to the highest temporal side lobe magnitude. The side lobes are denoted in Fig. 2, subplot $d$.

The averages of these metrics for the focusing onto the various virtual sources created are computed to give their respective values for a very small source (a single focal spot is about 150 microns in diameter). The $2 \mathrm{D}$ space versus time data from each adjacent pair of virtual sources is summed to form a $1 \mathrm{~mm}$ sized source, from which the averages of each of the four metrics are computed. The $2 \mathrm{D}$ space versus time data from each set of three adjacent virtual sources are then summed and the averages of the metrics are again computed for a source of $2 \mathrm{~mm}$ extent. This process is repeated for all the possible source sizes, with each repetition of the process adding one more virtual source until the extended virtual source reached a size of $24 \mathrm{~mm}$. Thus the four metrics could be plotted versus the size of the extended virtual source. Figure 3 displays these results. Note that, for sources less than a half wavelength in size, while the magnitude of the focusing increases with increasing size of the virtual source, the magnitude of the fringe energy (energy at locations other than the focal location) must be equally increasing since both $\beta$ and $\gamma$ do not appreciable increase. However, when the source is larger than a half wavelength both $\beta$ and $\gamma$ decrease with increasing source size until these metrics level off when the source is a wavelength or larger. $\delta$ tends to steadily increase despite the size of the source.

\section{Results and discussion}

The magnitude of the radiation from a arbitrarily shaped vibrating source (or sensitivity of a receiving source), which is small compared to the wavelength of sound it is emitting, effectively does not depend on the angle at which it emanates from the transducer (or incidence angle for receivers). ${ }^{15}$ However, when the wavelength of sound approaches the size of the transducer (the transducer is then considered to be of a finite size), the transducer becomes increasingly directional. The magnitude dependence as a function of angle is commonly called directivity. The higher the directivity of the source, the smaller the radiation aperture is of the source and thus it becomes increasingly difficult for the TRM to detect radiated energy from the source, which either directly propagates from the source to the TRM, or is reflected off of scatterers and

(a)

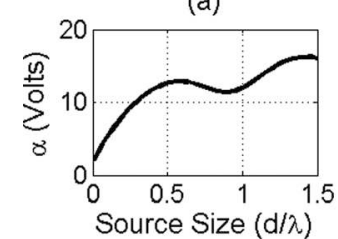

(b)

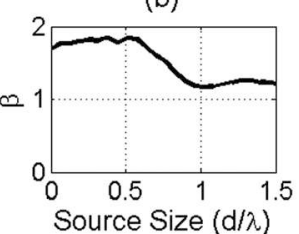

(c)

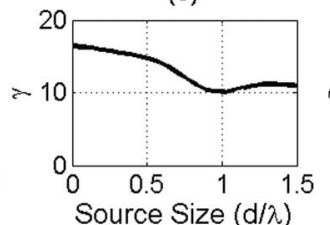

(d)

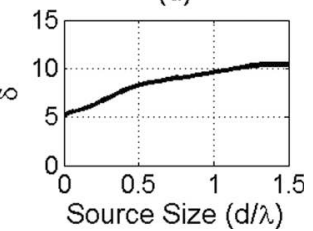

Fig. 3. Metrics quantifying the quality of the time reversal (TR) focusing on the virtual line source. (a) Peak magnitude of the spatial focus onto the line source. (b), (c), and (d) Peak magnitude from (a) divided by: the maximum fringe magnitude elsewhere, the average fringe magnitude elsewhere, and the peak side lobe magnitude of the focal signal, respectively. 
boundaries to the TRM. The quality of TR focusing should decrease with a smaller available source aperture. Therefore directional sources may be more difficult to reconstruct spatially than smaller sources which are generally more omnidirectional.

Several conclusions may be drawn from the results displayed in Fig. 3. The first conclusion is that the ability to spatially distinguish a TR focus appears to drop off when the source is larger than a half of a wavelength. From Fig. 3(b) it is apparent that $\beta$ approaches a value of 1.0 meaning that the magnitude of the TR focus is nearly equivalent to the magnitude of the nearby spatial energy. Thus if the source is one wavelength in length or larger, the focus is not easily distinguishable from other spatial energy, though the focal region may still be apparent with a source of this size since $\gamma$ is still at a value of about 10 [see Fig. 3(c)] and there is still a clear temporal focusing above the side lobes with $\delta \approx 10$ [see Fig. 3(d)].

\section{Conclusion}

This paper has experimentally characterized the ability of the TR process to reconstruct coherent sources of finite size. The experiments conducted in this work suggest that it becomes increasingly difficult for the TR process to reconstruct coherent sources as the size of the source increases past a half wavelength in size. Though conclusions have been drawn from experiments conducted in solid media, we believe that a similar result in fluid media should follow as well. These experiments are for a particular experimental setup and therefore the magnitude of the metrics computed may vary for other setups but we believe that the trends should hold.

\section{Acknowledgments}

This research was supported by institutional support (LDRD) at the Los Alamos National Laboratory. The authors wish to thank Robert Guyer, Carène Larmat and Pierre-Yves Le Bas for their helpful discussions and insights.

\section{References and links}

${ }^{1}$ M. Fink, "Time reversed acoustics," Phys. Today 50, 34-40 (1997).

${ }^{2}$ B. E. Anderson, M. Griffa, C. Larmat, T. J. Ulrich, and P. A. Johnson, "Time reversal," Acoust. Today 4(1), 5-16 (2008).

${ }^{3}$ T. J. Ulrich, P. A. Johnson, and A. M. Sutin, "Imaging nonlinear scatterers applying the time reversal mirror," J. Acoust. Soc. Am. 119(3), 1514-1518 (2006).

${ }^{4}$ T. J. Ulrich, P. A. Johnson, and R. A. Guyer, "Interaction dynamics of elastic waves with a complex nonlinear scatterer through the use of a time reversal mirror," Phys. Rev. Lett. 98, 104301 (2007).

${ }^{5}$ J. Brum, S. Catheline, N. Benech, and C. Negreira, "Shear elasticity estimation from surface wave: The time reversal approach,” J. Acoust. Soc. Am. 124(6), 3377-3380 (2008).

${ }^{6}$ A. Sutin, B. Libbey, L. Fillinger, and A. Sarvazyan, "Wideband nonlinear time reversal seismo-acoustic method for landmine detection," J. Acoust. Soc. Am. 125(4), 1906-1910 (2009).

${ }^{7}$ S. Dos Santos and Z. Prevorovsky, "Imaging of human tooth using ultrasound based chirp-coded nonlinear time reversal acoustics," Ultrasonics 51(6), 667-674 (2011).

${ }^{8}$ C. Larmat, J.-P. Montagner, M. Fink, Y. Capdeville, A. Tourin, and E. Clevede, "Time-reversal imaging of seismic sources and applications to the great Sumatra earthquake," Geophys. Res. Lett. 33, L19312 (2006).

${ }^{9}$ C. Larmat, R. Guyer, and P. A. Johnson, "Time-reversal methods in geophysics," Phys. Today 63, $31-35$ (2010).

${ }^{10}$ M. R. Bai and Y. K. Tsai, "Impact localization combined with haptic feedback for touch panel applications based on the time-reversal approach,” J. Acoust. Soc. Am. 129(3), 1297-1305 (2011)

${ }^{11}$ R. K. Ing, N. Quieffin, S. Catheline, and M. Fink, "In solid localization of finger impacts using acoustic time-reversal process" Appl. Phys. Lett. 87, 204104 (2005).

${ }^{12}$ M. Griffa, B. E. Anderson, R. A. Guyer, T. J. Ulrich, and P. A. Johnson, "Investigation of the robustness of time reversal acoustics in solid media through the reconstruction of temporally symmetric sources,” J. Phys. D: Appl. Phys. 41, 085415 (2008).

${ }^{13}$ S. Kremers, A. Fichtner, G. B. Brietzke, H. Igel, C. Larmat, L. Huang, and M. Käser, "Exploring the potentials and limitations of the time-reversal imaging of finite seismic sources," Solid Earth 2, 95-105 (2011). 
${ }^{14}$ T. J. Ulrich, M. Griffa, and B. E. Anderson, "Symmetry-based imaging condition in time reversed acoustics,” J. Appl. Phys. 104(6), 064912 (2008).

${ }^{15}$ L. E. Kinsler, A. R. Frey, A. B. Coppens, and J. V. Sanders, Fundamentals of Acoustics, 4th ed., 172-176 (Wiley, New York, 2000), pp. 171-204. 